Background and aim Timely and accurate diagnosis of children with osteomyelitis is crucial, diagnostic imaging play a major role in determine the presence of acute osteomyelitis, treatment planning and follow up. Physicians encouraged to take the advantage of all available modalities as early intervention would prevent all possible adverse outcome of late diagnosis . The aim of this study is to explore the different imaging modalities in verifying the diagnosis of paediatrics acute osteomyelitis.

Methods All cases diagnosed with Acute Osteomyelitis between January 2000 and December 2013 were retrospectively reviewed at main tertiary children hospital. Our approach included a detailed description of radiological features of paediatric patients with acute haematogenous osteomyelitis.

Results 79 cases of acute osteomyelitis were diagnosed. 68 (86.1\%) of children had X-Ray within first two weeks. $(51.5 \%)$ reported as normal compared to $(48.5 \%)$ abnormal (Periosteal reaction- Ostelytic lesions- soft tissue swelling). Ultrasound done in $34(43 \%)$ of children, (70.6\%) reported normal vs. $(29.4 \%)$ abnormal (effusion). MRI study done in 73 (92.4\%) and revealed osteomyelitis in $100 \%$ of imaging. 16 patients $(20.3 \%)$ had Bone Scan,(12.5\%) reported normal compared to $(87.5 \%)$ abnormal. (100\%) of children with positive bone scan had similar osteomyelitis on MRI.

Conclusion Our study confirmed that MRI is the gold standard of imaging modality which combines high sensitivity with specificity to confirm osteomyelitis in children despite having normal X-ray, Ultrasound and Bone scan. Simple X-ray might be useful to diagnose osteomyelitis if MRI is difficult to perform or if the cost plays a major role in the patient care.

\section{PO-0241 BILATERAL BASAL GANGLIA INFARCTION IN PNEUMOCOCCAL MENINGOENCEPHALITIS IN A CHILD}

C Neeleman, R Eijk. Intensive Care, Radboud University Medical Centre, Nijmegen, Netherlands

\subsection{6/archdischild-2014-307384.892}

Background and aims Basal ganglia infarction is considered a complication of chronic refractory meningitis. In acute infection the basal ganglia are usually spared. Here we report an exceptional case of bilateral infarction of the basal ganglia in a child with acute $S$. pneumoniae meningoencephalitis.

Methods A 4 year old girl with a short history of fever and left sided otalgia presented with drowsiness in a referral hospital. Cerebral spinal fluid (CSF) examination showed pleocytosis (900 leucocytes $/ \mathrm{mm}^{3}$ )and immediately corticosteroids, ceftriaxone and acyclovir were started. Because of a rapid decline inconsciousness she was transferred to our tertiairy PICU centre.

Results Ad admission the girl had become unresponsive and was intubated. Her pupils were mid wide and non-reacting to light. Neurologic examination showed a bipyramidal syndrome with hypertonicity of the lower extremities, brisk deep tendon reflexes and bilateral positive Babinski's. S.pneumoniae was cultured from CSF and blood. An MRI 4 days after admission showed bilateral sharply demarcated areas of high-signal intensity in the thalamus indicating infarction (Figure 1). In addition osteomyelitis of the tip of the petrous pyramid was observed. Her consciousness gradually improved with bilateral reactive pupils and spontaneous limb movements. Brainstem evoked response audiometry (BERA) of the left ear was negative. Gross motor deficits and impaired eye movements persisted.

Conclusion Acute pneumococcal meningoencephalitis can cause bilateral basal ganglia infarction in a child.

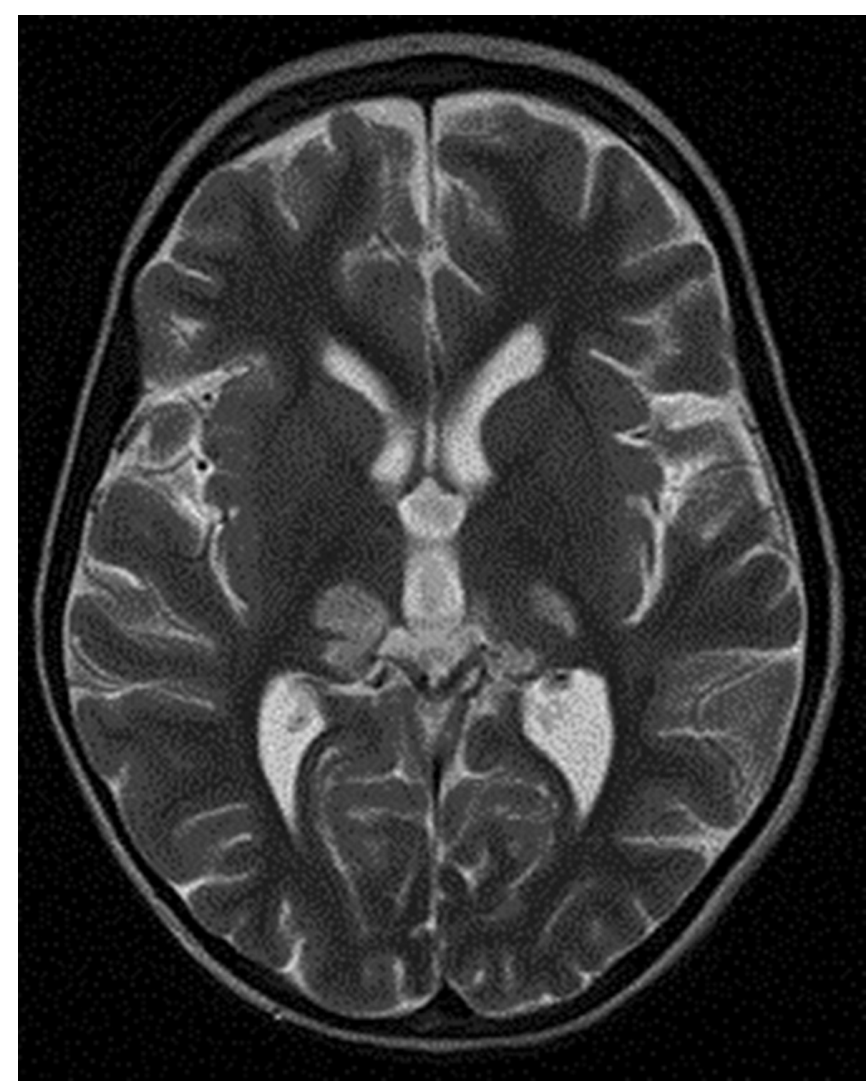

Abstract P0-0241 Figure 1 Magnetic resonance imaging (MRI) of the brain showing bilateral thalamic infarction

\section{PO-0242 PREVALENCE OF CONGENITAL TOXOPLASMOSIS IN NEWBORNS IN 2 EDUCATIONAL HOSPITALS IN TEHRAN IRAN}

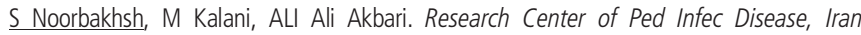
University of Medical Sciences, Tehran, Iran

\subsection{6/archdischild-2014-307384.893}

Background and objective Frequency and clinical manifestations of congenital toxoplasmosis in Iran is not determined, object of study was to determine the Frequency of positive serologic neonates for Toxoplasma from birth and follow up of them.

Methods In a cohort prospective study (2011-2012), Cord blood sample obtained from 270 neonates, toxoplasma serology tests $(\mathrm{IgG}, \operatorname{IgM})$ done, cases with positive toxo-IgM treated and followed

Finding Positive IgM and IgG determined 1.5\%, 44.1\% respectively. The most common manifestation was Eye (50\%) and brain $(50 \%)$.

Conclusion Early treatment of infected neonates and wide variation of toxoplasma infection in country is so important. Adding the toxoplasma serologic tests to neonatal screening test is needed and recommended.

\section{PO-0243 PROCALCITONIN USE IN DIAGNOSIS OF PAEDIATRIC MENINGITIS}

S Noorbakhsh, KH Shabanzadeh. Research Center of Ped Infec Disease, Iran University of Medical Sciences, Tehran, Iran

10.1136/archdischild-2014-307384.894 